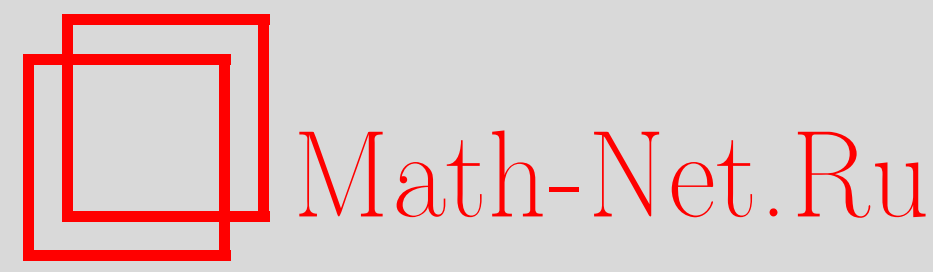

И. Д. Чуешов, Аналитичность глобальных аттракторов и определяющие узлы для некоторого класса нелинейных волновых уравнений с демпфированием, Матем. сб., 2000, том 191, номер 10, 119-136

DOI: https://doi.org/10.4213/sm519

Использование Общероссийского математического портала Math-Net.Ru подразумевает, что вы прочитали и согласны с пользовательским соглашением http://www.mathnet.ru/rus/agreement

Параметры загрузки:

IP : 54.224 .135 .184

26 апреля 2023 г., 14:02:31 


\title{
И. Д. Чуешов \\ Аналитичность глобальных аттракторов и определяющие узлы для некоторого класса нелинейных волновых уравнений с демпфированием
}

\begin{abstract}
Доказана регулярность по Жевре глобальных аттракторов динамических систем, порождаемых некоторым классом диссипативных систем связанных нелинейных волновых уравнений с периодическими граничньми условиями. Этот результат означает, что элементы аттрактора являются вещественно-аналитическими функциями по пространственным переменньм, и позволяет доказать существование двух определяющих узлов для задач с одной пространственной переменной.

Библиография: 15 названий.
\end{abstract}

\section{Введение}

Основная цель данной работы - показать, что некоторые результаты о регулярности по Жевре глобальных аттракторов полулинейных параболических уравнений (см., например, [1]-[5] и ссылки, приведенные в этих работах) остаются справедливыми для некоторого класса диссипативных нелинейных волновых уравнений. В качестве основной модели рассматривается следующая система связанных нелинейных волновых уравнений с демпфированием

$$
\begin{gathered}
\partial_{t}^{2} u_{j}(t, x)+\gamma \partial_{t} u_{j}(t, x)+\sum_{i=1}^{m} d_{i j}(-\Delta+1) u_{i}(t, x) \\
\quad+f_{j}\left(u_{1}(t, x), \ldots, u_{m}(t, x)\right)=h_{j}(x), \\
x \in \mathbb{R}^{n}, \quad t>0, \quad j=1, \ldots, m,
\end{gathered}
$$

где неизвестная векторная функция $u(x, t)=\left\{u_{1}(x, t), \ldots, u_{m}(x, t)\right\}, m \geqslant 1$, удовлетворяет периодическим граничньм условиям:

$$
u\left(t, x+L e_{k}\right)=u(t, x), \quad k=1, \ldots, n, \quad x \in \mathbb{R}^{n}, \quad t>0 .
$$

Здесь $\left\{e_{k}\right\}$ - стандартный базис в $\mathbb{R}^{n}, L$-положительная константа, $D=\left\{d_{i j}\right\}_{i, j=1}^{m}$ - положительная матрица, $\Delta$ - оператор Лапласа, $h(x)=\left\{h_{1}(x), \ldots, h_{m}(x)\right\}$ - заданная вещественно-аналитическая $L$-периодическая векторная функция. Снабдим задачу (1), (2) начальньми условиями

$$
u(0, x)=u_{0}(x), \quad \partial_{t} u(0, x)=u_{1}(x), \quad x \in \mathbb{R}^{n},
$$

где $u_{0}(x)$ и $u_{1}(x)-L$-периодические векторные функции. Предполагается, что $f_{j}(u) \equiv f_{j}\left(u_{1}, \ldots, u_{m}\right)$ - вешественно-аналитические функции относительно переменной $u=\left(u_{1}, \ldots, u_{m}\right)$.

(C) И. Д. Чуешов 2000 
В статье изучаются свойства асимптотической регулярности решений задачи (1)-(3). Основной результат (см. теорему 1.1) утверждает, что глобальньй аттрактор задачи принадлежит некоторому классу Жевре. Отсюда вытекает, что его элементы являются вещественно-аналитическими функциями по пространственным переменным. Аналогичный результат хорошо известен для некоторых полулинейных параболических уравнений (см., например, [1], [2]). Однако в параболическом случае метод доказательства сушественно опирается на регуляризируюший эффект параболических уравнений (решение в момент $t>0$ является более гладким, чем его начальное условие). Это явление позволяет доказать существование поглощающего множества, состоящего из вещественно-аналитических функций. Отсюда вытекает, что каждое решение после некоторого переходного интервала времени становится вешественно-аналитической функцией по пространственной переменной. Для случая эволюционных уравнений второго порядка по времени регуляризирующий эффект отсутствует (пространственная гладкость начальных условий сохраняется с течением времени). Для этих уравнений удается доказать лишь существование равномерно притягивающего множества, состоящего из вещественно-аналитических функций. Основным моментом здесь является построение подходящего разложения эволюционного оператора на убьвающую и компактную компоненты. Рассуждения опираются на некоторые идеи, представленные в [6] (см. также работу [7], в которой была установлена аналитичность аттрактора для слабо демпфированного нелинейного уравнения Шрёдингера с возбуждением). В качестве приложения теоремы 1.1 об аналитичности аттрактора задачи (1)-(3) в данной работе доказано также, что пространственно-одномерньй вариант этой задачи обладает двумя определяющими узлами, т.е. асимптотическое поведение решений полностью определяется их динамикой в двух точках, лежащих внутри пространственного интервала $(0, L)$. Отметим, что впервые связь между регулярностью по Жевре и существованием малого числа определяющих узлов была установлена в работе [3], посвященной уравнению Гинзбурга-Ландау (см. также [4], [7] и обзор [8], где приведены аналогичные результаты для других уравнений). В работе [8] содержится также общее обсуждение проблемы существования определяющих функционалов для бесконечномерных уравнений.

Статья построена следующим образом. В 11 вводятся обозначения, дается некоторое описание классов Жевре и формулируются основные результаты. Параграф 2 посвящен доказательству теоремы о регулярности по Жевре аттрактора динамической системы, порождаемой задачей (1)-(3). В 33 установлено существование двух определяющих узлов в случае одной пространственной переменной. В $\S 4$ приведено несколько примеров.

\section{§1. Предварительные рассмотрения и формулировка основных результатов}

Пусть $\Omega=(0, L)^{n} \equiv(0, L) \times \cdots \times(0, L) \subset \mathbb{R}^{n}$. Для каждого $s \geqslant 0$ рассмотрим соболевское пространство

$$
H_{\mathrm{per}}^{s}(\Omega)=\left\{u(x) \in H_{\mathrm{loc}}^{s}\left(\mathbb{R}^{n}\right): u\left(x+L e_{j}\right)=u(x), j=1, \ldots, n, x \in \mathbb{R}^{n}\right\} .
$$

Так как $-\Delta$ порождает неотрицательный самосопряженный оператор в пространстве $L^{2}(\Omega)$ с областью определения $\mathbf{D}(-\Delta)=H_{\mathrm{per}}^{2}(\Omega)$, то можно определить положительный оператор $A=(-\Delta+1)^{1 / 2}$ на области определения $\mathbf{D}(A)=H_{\mathrm{per}}^{1}(\Omega)$ 
и задать в пространстве $H_{\mathrm{per}}^{s}(\Omega)$ скалярное произведение

$$
(u, v)_{s}=\int_{\Omega}\left((-\Delta+1)^{s} u\right)(x) v(x) d x=\left(A^{s} u, A^{s} v\right), \quad s \geqslant 0
$$

где $(\cdot, \cdot)$ - скалярное произведение в $L^{2}(\Omega)$. Ниже символом $\|\cdot\|_{s}$ обозначается соответствующая норма в $H_{\text {per }}^{s}(\Omega)$, а символом $\|\cdot\|$ - норма в $L^{2}(\Omega)$. Отметим, что каждый элемент $u(x) \in H_{\text {per }}^{s}(\Omega)$ может быть представлен в виде

$$
u(x)=\sum_{j \in \mathbb{Z}^{n}} u_{j} \exp \left\{i(j, x) \frac{2 \pi}{L}\right\}
$$

где коэффициенты Фурье $u_{j}$ обладают свойством $\bar{u}_{j}=u_{-j}$ (черта обозначает комплексное сопряжение) и

$$
\|u\|_{s}^{2}=L^{n} \sum_{j \in \mathbb{Z}^{n}}\left(1+(2 \pi / L)^{2} \cdot|j|^{2}\right)^{s}\left|u_{j}\right|^{2}<\infty
$$

В дальнейшем для каждого $N=1,2, \ldots$ будем обозначать через $P_{N}$ ортопроектор в $L^{2}(\Omega)$ на подпространство

$$
L_{N}=\left\{u \in L^{2}(\Omega): u(x)=\sum_{|j| \leqslant N} u_{j} \exp \left\{i(j, x) \frac{2 \pi}{L}\right\}, \bar{u}_{j}=u_{-j}\right\} .
$$

Введем теперь классы Жевре $G_{\sigma}^{s}(\Omega)$ формулой $G_{\sigma}^{s}(\Omega)=\mathbf{D}\left(A^{s} e^{\sigma A}\right)$, где, как и ранее, $A=(-\Delta+1)^{1 / 2}$ с периодическими граничньми условиями на $\Omega$, а $\mathbf{D}(B)$ обозначает область определения оператора $B$. Здесь $s \geqslant 0$ и $\sigma \geqslant 0$ - некоторые константы. Эти классы являются гильбертовыми пространствами, состоящими из функций вида (1.1) таких, что

$$
\begin{aligned}
|u|_{G_{\sigma}^{s}(\Omega)}^{2} & =\left\|A^{s} \exp \{\sigma A\} w\right\|^{2} \\
& \equiv L^{n} \sum_{j \in \mathbb{Z}^{n}}\left(1+(2 \pi / L)^{2}|j|^{2}\right)^{s} \exp \left\{2 \sigma\left(1+(2 \pi / L)^{2}|j|^{2}\right)^{1 / 2}\right\}\left|u_{j}\right|^{2}<\infty
\end{aligned}
$$

Символом $(u, v)_{G s(\Omega)}$ будем обозначать соответствующие скалярные произведения. Ясно, что $G_{0}^{s}(\Omega)=H_{\text {per }}^{s}(\Omega)$ для любого $s \geqslant 0$ и $G_{\sigma}^{s}(\Omega) \subset H_{\mathrm{per}}^{m}(\Omega)$ для всех $s \geqslant 0, \sigma>0$ и $m \geqslant 0$. Более того, каждый элемент $v(x) \in G_{\sigma}^{s}(\Omega), \sigma>0$, может быть продолжен как аналитическая функция в параллелепипед

$$
\Pi^{n}=\left\{z \in \mathbb{C}^{n}: \operatorname{Re} z \in \Omega, \operatorname{Im} z \in(-\sigma, \sigma)^{n}\right\}
$$

В дальнейшем понадобится следуюшая лемма (ее доказательство см. в [5]).

ЛЕмма 1.1. Пусть $s>n / 2, \sigma \geqslant 0$ и предположимм, что функиии и $(x)$ и $v(x)$ лежат в классе $G_{\sigma}^{s}(\Omega)$. Тогда $u(x) \cdot v(x) \in G_{\sigma}^{s}(\Omega)$ и существует константа $C_{s}$, не зависящая от $\sigma$ и такая, что

$$
|u \cdot v|_{G_{\sigma}^{s}(\Omega)} \leqslant C_{s} \cdot|u|_{G_{\sigma}^{s}(\Omega)} \cdot|v|_{G_{\sigma}^{s}(\Omega)} .
$$


Ниже будут также рассматриваться векторные классы $\mathbf{H}^{s}(\Omega)$ и $\mathbf{G}_{\sigma}^{s}(\Omega)$, определяемые формулами

$$
\mathbf{H}_{\mathrm{per}}^{s}(\Omega) \equiv\left[H_{\mathrm{per}}^{s}(\Omega)\right]^{m}=\left\{u(x) \equiv\left(u_{1}, \ldots, u_{m}\right): u_{j} \in H_{\mathrm{per}}^{s}(\Omega), j=1, \ldots, m\right\}
$$

и

$$
\mathbf{G}_{\sigma}^{s}(\Omega) \equiv\left[G_{\sigma}^{s}(\Omega)\right]^{m}=\left\{u(x) \equiv\left(u_{1}, \ldots, u_{m}\right): u_{j} \in G_{\sigma}^{s}(\Omega), j=1, \ldots, m\right\}
$$

с нормами

$$
\|u\|_{s}^{2}=\sum_{j=1}^{m}\left\|u_{j}\right\|_{s}^{2} \quad \text { и }|u|_{G_{\sigma}^{s}(\Omega)}^{2}=\sum_{j=1}^{m}\left|u_{j}\right|_{G_{\sigma}^{s}(\Omega)}^{2} .
$$

Ради простоты сохраняются те же самые символы для норм и скалярных произведений в $\mathbf{H}_{\mathrm{per}}^{s}(\Omega)$ и $H_{\mathrm{per}}^{s}(\Omega)$ и в $\mathbf{G}_{\sigma}^{s}(\Omega)$ и $G_{\sigma}^{s}(\Omega)$.

Перепишем уравнения (1) как задачу в пространстве $\mathbf{L}^{2}(\Omega)$ вида

$$
\partial_{t}^{2} u(t, x)+\gamma \partial_{t} u(t, x)+\mathscr{L} u(t, x)+f(u(t, x))=h(x), \quad x \in \mathbb{R}^{n}, \quad t>0
$$

Здесь $f(u)$ и $h(x)$ - векторные функции вида

$$
f(u)=\left(f_{1}\left(u_{1}, \ldots, u_{m}\right), \ldots, f_{m}\left(u_{1}, \ldots, u_{m}\right)\right), \quad h=\left(h_{1}, \ldots, h_{m}\right),
$$

а $\mathscr{L}$ - положительный оператор в $\mathbf{L}^{2}(\Omega)$ с областью определения $\mathbf{D}(\mathscr{L})=\mathbf{H}_{\mathrm{per}}^{2}(\Omega)$, задаваемый формулой

$$
(\mathscr{L} u)_{j}(x)=\sum_{i=1}^{m} d_{i j} A^{2} u_{i}(x), \quad j=1, \ldots, m, \quad u(x)=\left(u_{1}, \ldots, u_{m}\right) \in \mathbf{H}_{\mathrm{per}}^{2}(\Omega),
$$

где $D=\left\{d_{i j}\right\}_{i, j=1}^{m}-$ положительная матрица такая, что для некоторых $0<d_{0} \leqslant d_{1}$ справедлива оценка

$$
d_{0} \cdot \sum_{i=1}^{m} \xi_{i}^{2} \leqslant \sum_{i, j=1}^{m} d_{i j} \xi_{i} \xi_{j} \leqslant d_{1} \cdot \sum_{i=1}^{m} \xi_{i}^{2} \text { для всех } \xi=\left(\xi_{1}, \ldots, \xi_{m}\right) \in \mathbb{R}^{m}
$$

Относительно задачи (1.6), (2), (3) предполагаются выполненными следующие условия.

(A1) При некотором неотрицательном $s>n / 2-1$ задача $(1.6),(2),(3)$ корректно разрешима в классе

$$
\mathscr{W}_{s}=C^{1}\left(0,+\infty ; \mathbf{H}_{\mathrm{per}}^{s}(\Omega)\right) \cap C\left(0,+\infty ; \mathbf{H}_{\mathrm{per}}^{s+1}(\Omega)\right)
$$

(А2) Эволюционньй оператор $S_{t}$, порождаемьй в пространстве $\mathbf{E}_{s}=\mathbf{H}_{\mathrm{per}}^{s+1}(\Omega) \times$ $\mathbf{H}_{\text {per }}^{s}(\Omega)$ формулой $S_{t}\left(u_{0} ; u_{1}\right)=\left(u(t) ; \partial_{t} u(t)\right)$, где $u(t)-$ решение задачи $(1.6),(2),(3)$, обладает глобальньм аттрактором $\mathscr{A}$, ограниченным в пространстве $\mathbf{E}_{s}$, т.е. (см. [9]-[13]) сушествует ограниченное замкнутое множество $\mathscr{A}$ в $\mathbf{E}_{s}$ такое, что $S_{t} \mathscr{A}=\mathscr{A}$ для всех $t \geqslant 0$ и

$$
\lim _{t \rightarrow+\infty} \sup \left\{\operatorname{dist}_{\mathbf{E}_{s}}\left(S_{t} y, \mathscr{A}\right): y \in B\right\}=0
$$


для любого ограниченного множества $B$ из $\mathbf{E}_{s}$.

(А3) Функция $h(x)=\left\{h_{1}(x), \ldots, h_{m}(x)\right\}$ лежит в классе $\mathbf{G}_{\sigma_{0}}^{s}(\Omega)$ при некотором $\sigma_{0}>0$ и для каждого $j=1, \ldots, m$ функция $f_{j}(u)=f_{j}\left(u_{1}, \ldots, u_{m}\right)$ может быть представлена в виде

$$
f_{j}(u)=\sum_{\alpha} b_{\alpha}^{(j)} u^{\alpha}
$$

Здесь $\alpha=\left(\alpha_{1}, \ldots, \alpha_{m}\right) \in \mathbb{Z}_{+}^{m}$ - мультииндекс такой, что

$$
n(\alpha) \equiv \alpha_{1}+\cdots+\alpha_{m} \geqslant 1, \quad u^{\alpha}=u_{1}^{\alpha_{1}} \cdot \ldots \cdot u_{m}^{\alpha_{m}},
$$

а коэффициенты $\left\{b_{\alpha}^{(j)}\right\}$ обладают свойством

$$
g_{j}(r) \equiv \sum_{k=1}^{\infty} r^{k}\left(\sum_{n(\alpha)=k}\left|b_{\alpha}^{(j)}\right|\right)<\infty
$$

для всех $0<r<r_{0}$ и $j=1, \ldots, m$, где $r_{0}$ - достаточно большое число.

Примеры задач, для которых выполнены предположения (A1)-(А3), приведены в $\oint 4$ (см. также [10] и [12]).

ЗАмЕчАНИЕ 1.1. Отметим, что при условиях (A1) и (А3) задача (1.6), (2), (3) корректно разрешима в каждом классе

$$
\mathscr{W}_{s+k}=C^{1}\left(0,+\infty ; \mathbf{H}_{\mathrm{per}}^{s+k}(\Omega)\right) \cap C\left(0,+\infty ; \mathbf{H}_{\mathrm{per}}^{s+k+1}(\Omega)\right), \quad k=0,1, \ldots
$$

Кроме того, каждое пространство $\mathbf{E}_{s+k}=\left(\mathbf{H}_{\text {per }}^{s+k+1} \times \mathbf{H}_{\text {per }}^{s+k}\right)(\Omega)$ является инвариантным относительно эволюционного оператора $S_{t}$, так как $S_{t} \mathbf{E}_{s+k}=\mathbf{E}_{s+k}$, и если имеет место $(\mathrm{A} 2)$, глобальный аттрактор $\mathscr{A}$ лежит в каждом из пространств $\mathbf{E}_{s+k}, k=0,1, \ldots$. Следовательно, $\mathscr{A} \subset C_{\mathrm{per}}^{\infty}(\Omega) \times C_{\mathrm{per}}^{\infty}(\Omega)$. Для доказательства этих фактов нужно повторить рассуждения, приведенные в [14]. Здесь не приводится подробных доказательств, потому что эти свойства не используются в последующих рассмотрениях. Отметим также, что в случае $m=1$ задача (1.6), (2), (3) обладает функцией Ляпунова (см., например, [10], [11]) и поэтому глобальный аттрактор $\mathscr{A}$ имеет регулярную структуру, т.е. состоит из неподвижных точек и их неустойчивых многообразий (см. [10]).

Основным результатом работы является следующая теорема.

Теорема 1.1. Предположим, что выполняются условия (A1)-(А3). Тогда существует $0<\sigma \leqslant \sigma_{0}$ такое, что глобальный аттрактор $\mathscr{A}$ динамической системь $\left(S_{t}, \mathbf{E}_{s}\right)$, порождаемой задачей $(1)-(3)$, лежит в $\mathbf{G}_{\sigma}^{s+1}(\Omega) \times \mathbf{G}_{\sigma}^{s}(\Omega)$ $u$

$$
\sup \left\{\left|u_{0}\right|_{G_{\sigma}^{s+1}(\Omega)}^{2}+\left|u_{1}\right|_{G_{\sigma}^{s}(\Omega)}^{2}:\left(u_{0}, u_{1}\right) \in \mathscr{A}\right\}<\infty .
$$

Таким образом, все элементы глобального аттрактора являются вещественно-аналитическими функциями по пространственным переменнып.

Теорема 1.1 позволяет также доказать следуюшее утверждение о сушествовании двух определяющих узлов для задачи (1)-(3) в случае одной пространственной переменной. 
ТЕОРема 1.2. Предположим, что $n=1$ и условия (A1)-(А3) выполняются при $s=0$. Пусть $x_{1}$ и $x_{2}-$ два узла таких, что $0 \leqslant x_{1}<x_{2} \leqslant L$. Пусть $u(t)=\left(u_{1}(t, x), \ldots, u_{m}(t, x)\right) u u^{*}(t)=\left(u_{1}^{*}(t, x), \ldots, u_{m}^{*}(t, x)\right)-$ решения задачи (1), (2) при $n=1$ из класса $\mathscr{W}_{2}$. Тогда существует $\Delta_{0}$, не зависящее от и и $u^{*}$, такое, что если $x_{2}-x_{1} \leqslant \Delta_{0}$, то из условия

$$
\lim _{t \rightarrow \infty} \max _{l=1,2} \sum_{j=1}^{m}\left|u_{j}\left(t, x_{l}\right)-u_{j}^{*}\left(t, x_{l}\right)\right|=0
$$

вытекает

$$
\lim _{t \rightarrow \infty}\left\{\left\|\partial_{t}\left(u(t)-u^{*}(t)\right)\right\|^{2}+\left\|u(t)-u^{*}(t)\right\|_{1}^{2}\right\}=0 .
$$

Теорема 1.2 означает, что асимптотическое поведение любого решения задачи (1)-(3) класса $\mathscr{W}_{2}$ полностью определяется значениями этого решения в любых двух достаточно близких друг к другу узлах.

\section{§2. Регулярность аттрактора по Жевре}

В этом параграфе доказана теорема 1.1. Метод ее доказательства использует некоторые идеи, представленные в [6] и [7] для нелинейного уравнения Шрёдингера. Ниже предполагается выполнение всех условий, приведенных в 1 .

Пусть $u(t)=\left(u_{1}(t), \ldots, u_{m}(t)\right)$ - решение задачи $(1.6),(2),(3)$ с начальными условиями $y=\left(u_{0} ; u_{1}\right) \in B$, где $B$ - какое-либо ограниченное множество в $\mathbf{E}_{s}$. Из условия (А2) о сушествовании глобального аттрактора вытекает, что найдется $t_{0}=t_{0}(B)$ такое, что

$$
\sup _{y \in B}\left\{\left\|\partial_{t} u(t)\right\|_{s}^{2}+\|u(t)\|_{s+1}^{2}\right\} \leqslant R_{0}^{2} \text { для всех } t \geqslant t_{0}=t_{0}(B),
$$

где $R_{0}=1+\sup _{y \in \mathscr{A}}\|y\|_{\mathbf{E}_{s}}$. Пусть $p_{N}(t)=P_{N} u(t) \equiv\left(P_{N} u_{1}(t), \ldots, P_{N} u_{m}(t)\right)$, где $P_{N}$ - ортопроектор на подпространство $L_{N}$, определяемое формулой (1.2). Рассмотрим в пространстве $Q_{N} \mathbf{L}^{2}(\Omega)$, где $Q_{N}=1-P_{N}$, следующую вспомогательную задачу

$$
\partial_{t}^{2} w(t, x)+\gamma \partial_{t} w(t, x)+\mathscr{L} w(t, x)+Q_{N} f\left(p_{N}(t)+w(t, x)\right)=Q_{N} h(x)
$$

в классе периодических функций:

$$
w\left(t, x+L e_{k}\right)=w(t, x), \quad k=1, \ldots, n, \quad x \in \mathbb{R}^{n}, \quad t>t_{0}=t_{0}(B),
$$

с нулевьми начальными условиями в момент $t_{0}=t_{0}(B)$ :

$$
w\left(t_{0}, x\right)=0, \quad \partial_{t} w\left(t_{0}, x\right)=0, \quad x \in \mathbb{R}^{n} .
$$

Отметим, что уравнение (2.2) формально получается как проекция уравнения (1.6) на подпространство $Q_{N} \mathbf{L}^{2}(\Omega)$. Однако из-за нулевых начальных условий для $w(t)$ в момент $t_{0}$ функция $v(t)=p_{N}(t)+w(t)$ не обязательно является решением исходной задачи (1)-(3).

В приведенных ниже двух леммах будет показано, что решение $w(t)$ задачи (2.2)-(2.4) по пространственным переменным принадлежит классу Жевре и аппроксимирует решение $u(t)$ исходной задачи при $t \rightarrow \infty$. Эти свойства дают возможность доказать существование равномерно притягиваюшего ограниченного в 
некотором классе Жевре множества и воспользоваться стандартными результатами об аттракторах асимптотически компактных динамических систем (см., например, [9], [11]).

Лемма 2.1. Существуют числа $0<\sigma \equiv \sigma_{N} \leqslant \sigma_{0}, N_{0} \geqslant 1 u R>0$, не зависящее от $N$, такие, что задача (2.2)-(2.4) имеет единственное решение в классе

$$
C^{1}\left(t_{0},+\infty ; Q_{N} \mathbf{G}_{\sigma}^{s}(\Omega)\right) \cap C\left(t_{0},+\infty ; Q_{N} \mathbf{G}_{\sigma}^{s+1}(\Omega)\right), \quad N \geqslant N_{0}
$$

где $Q_{N}=1-P_{N}$, а $P_{N}$ - ортопроектор на подпространство $L_{N}$, определяемое с помощью (1.2). Это решение обладает свойством

$$
\left|\partial_{t} w(t)\right|_{G_{\sigma}^{s}(\Omega)}^{2}+|w(t)|_{G_{\sigma}^{s+1}(\Omega)}^{2} \leqslant R^{2} \quad \partial \Omega_{\text {я }} \quad \text { всех } \quad t \geqslant t_{0}=t_{0}(B), \quad N \geqslant N_{0} .
$$

ДокаЗАтельство. Рассмотрим в пространстве $Q_{M, N} \mathbf{L}^{2}(\Omega)$, где $Q_{M, N}=$ $P_{M}-P_{N}$ с $M>N$, вместо (2.2) следуюшее уравнение

$$
\partial_{t}^{2} w(t, x)+\gamma \partial_{t} w(t, x)+\mathscr{L} w(t, x)+Q_{M, N} f\left(p_{N}(t)+w(t, x)\right)=Q_{M, N} h(x) .
$$

Ясно, что это уравнение имеет единственное решение

$$
w_{M}(t, x)=\sum_{N \leqslant|j| \leqslant M} w_{j}(t) \exp \left\{i(j, x) \frac{2 \pi}{L}\right\}, \quad w_{M}\left(t_{0}, x\right)=0
$$

на некотором интервале $\left(t_{0}, t_{0}+T_{M, N}\right)$. Если будет доказана равномерная оценка (2.5) для $w_{M}(t)$ на интервале сушествования $\left(t_{0}, t_{0}+T_{M, N}\right)$, то можно будет сделать вывод о возможности продолжения этого решения на полуось $\left(t_{0},+\infty\right)$. После этого можно выполнить предельный переход $M \rightarrow \infty$ и установить сушествование решения для (2.2)-(2.4) со свойством (2.5). Легко обнаружить, что это решение единственно. Таким образом, нужно лишш доказать для $w_{M}(t)$ равномерную оценку (2.5) на любом интервале существования. Ниже для краткости индекс $M$ будем опускать.

При $\nu>0$ и $0<\sigma \leqslant \sigma_{0}$ определим функционал

$$
V(t) \equiv V\left(\partial_{t} w(t), w(t)\right)=V_{0}\left(\partial_{t} w(t), w(t)\right)+\nu \cdot V_{1}\left(\partial_{t} w(t), w(t)\right)
$$

где

$$
V_{0}\left(\partial_{t} w(t), w(t)\right)=\frac{1}{2}\left(\left|\partial_{t} w(t)\right|_{G_{\sigma}^{s}(\Omega)}^{2}+\sum_{i, j=1}^{m} d_{i j}\left(w_{i}(t), w_{j}(t)\right)_{G_{\sigma}^{s+1}(\Omega)}\right)
$$

И

$$
V_{1}\left(\partial_{t} w(t), w(t)\right)=\left(w(t), \partial_{t} w(t)\right)_{G_{\sigma}^{s}(\Omega)}+\frac{\gamma}{2}|w(t)|_{G_{\sigma}^{s}(\Omega)}^{2}
$$

Ясно, что для каждого $\nu \leqslant \gamma / 2$ справедливо соотношение

$$
\beta_{1}\left(\left|\partial_{t} w\right|_{G_{\sigma}^{s}(\Omega)}^{2}+|w|_{G_{\sigma}^{s+1}(\Omega)}^{2}\right) \leqslant V(t) \leqslant \beta_{2}\left(\left|\partial_{t} w\right|_{G_{\sigma}^{s}(\Omega)}^{2}+|w|_{G_{\sigma}^{s+1}(\Omega)}^{2}\right)
$$


с некоторыми положительными константами $\beta_{j}$, не зависящими от $\nu$. Стандартные вычисления дают, что

$\frac{d}{d t} V_{0}\left(\partial_{t} w(t), w(t)\right)=-\gamma\left|\partial_{t} w(t)\right|_{G_{\sigma}^{s}(\Omega)}^{2}+\left(-Q_{N} f\left(p_{N}(t)+w(t)\right)+Q_{N} h, \partial_{t} w(t)\right)_{G_{\sigma}^{s}(\Omega)}$.

Поэтому

$$
\frac{d}{d t} V_{0}\left(\partial_{t} w, w\right) \leqslant-\frac{\gamma}{2}\left|\partial_{t} w\right|_{G_{\sigma}^{s}(\Omega)}^{2}+\gamma^{-1}\left|Q_{N} f\left(p_{N}(t)+w\right)\right|_{G_{\sigma}^{s}(\Omega)}^{2}+\gamma^{-1}|h|_{G_{\sigma}^{s}(\Omega)}^{2} .
$$

Мы также имеем, что

$$
\begin{aligned}
\frac{d}{d t} V_{1}\left(\partial_{t} w, w\right) \leqslant\left|\partial_{t} w\right|_{G_{\sigma}^{s}(\Omega)}^{2}-d_{0}|w|_{G_{\sigma}^{s+1}(\Omega)}^{2} & \\
& +\frac{1}{2}\left|Q_{N} f\left(p_{N}(t)+w\right)\right|_{G_{\sigma}^{s}(\Omega)}^{2}+|w|_{G_{\sigma}^{s}(\Omega)}^{2}+\frac{1}{2}|h|_{G_{\sigma}^{s}(\Omega)}^{2}
\end{aligned}
$$

Из (2.6)-(2.8) вытекает сушествование $\nu>0$ и $\mu>0$ таких, что

$$
\begin{aligned}
\frac{d}{d t} V(t)+\mu V(t) & \leqslant-\frac{\nu d_{0}}{2}|w|_{G_{\sigma}^{s+1}(\Omega)}^{2} \\
& +C_{1} \cdot\left|Q_{N} f\left(p_{N}(t)+w\right)\right|_{G_{\sigma}^{s}(\Omega)}^{2}+C_{2} \cdot|w|_{G_{\sigma}^{s}(\Omega)}^{2}+C_{3} \cdot|h|_{G_{\sigma}^{s}(\Omega)}^{2},
\end{aligned}
$$

где константы $C_{j}>0$ не зависят от $N$. Так как

$$
|w|_{G_{\sigma}^{s}(\Omega)}^{2} \leqslant\left(1+N^{2}\right)^{-\left(s^{*}-s\right)} \cdot|w|_{G_{\sigma}^{s^{*}}(\Omega)}^{2}, \quad w \in Q_{N} G_{\sigma}^{s^{*}}(\Omega), \quad s<s^{*}
$$

TO

$$
\frac{d}{d t} V(t)+\mu V(t) \leqslant-\frac{\nu d_{0}}{4}|w|_{G_{\sigma}^{s+1}(\Omega)}^{2}+C_{1} \cdot\left|Q_{N} f\left(p_{N}(t)+w\right)\right|_{G_{\sigma}^{s}(\Omega)}^{2}+C_{2}
$$

для всех достаточно больших $N$, где константы $C_{j}>0$ не зависят от $N, \sigma \leqslant \sigma_{0}$.

Оценим теперь величину $\left|Q_{N} f\left(p_{N}(t)+w\right)\right|_{G_{\sigma}^{s}(\Omega)}^{2}$, опираясь на представление $f_{j}\left(p_{N}(t)+w\right)$ в виде

$$
f_{j}\left(p_{N}+w\right)=f_{j}\left(p_{N}\right)+\left(\nabla f_{j}\left(p_{N}\right), w\right)+\int_{0}^{1}(1-\tau)\left(\nabla^{2} f_{j}\left(p_{N}+\tau w\right) w, w\right) d \tau
$$

где

$$
\left(\nabla f_{j}, w\right)=\sum_{k=1}^{m} \partial_{u_{k}} f_{j} \cdot w_{k} \quad \text { и }\left(\nabla^{2} f_{j} w, w\right)=\sum_{k, l=1}^{m} \partial_{u_{k} u_{l}}^{2} f_{j} \cdot w_{k} \cdot w_{l} .
$$

Пусть $s^{*}$ - любое число, обладающее свойством $\max \{n / 2, s\}<s^{*}<s+1$. Из леммы 1.1 вытекает, что

$$
\begin{aligned}
\left|f\left(p_{N}(t)\right)\right|_{G_{\sigma}^{s^{*}}(\Omega)}^{2} & =\sum_{j=1}^{m}\left|f_{j}\left(p_{N}(t)\right)\right|_{G_{\sigma}^{s^{*}}(\Omega)}^{2} \\
& \leqslant \sum_{j=1}^{m}\left(\sum_{\alpha}\left|b_{\alpha}^{(j)}\right| \cdot\left|\left(p_{N}(t)\right)^{\alpha}\right|_{G_{\sigma}^{s^{*}}(\Omega)}\right)^{2} \\
& \leqslant \sum_{j=1}^{m}\left(\sum_{\alpha}\left|b_{\alpha}^{(j)}\right| \cdot C_{s^{*}}^{n(\alpha)-1} \cdot \prod_{i=1}^{m}\left|\left(p_{N}(t)\right)_{i}\right|_{G_{\sigma}^{s^{*}}(\Omega)}^{\alpha_{i}}\right)^{2} \\
& \leqslant C_{s^{*}}^{-2} \cdot \sum_{j=1}^{m}\left[g_{j}\left(C_{s^{*}}\left|p_{N}(t)\right|_{G_{\sigma}^{s^{*}}(\Omega)}\right)\right]^{2}
\end{aligned}
$$


где функции $g_{j}(r)$ определяются соотношениями $(1.8)$, а $C_{s^{*}}-$ константа из леммы 1.1 c $s=s^{*}$. Аналогичным образом имеем, что

$$
\begin{aligned}
\left|\left(\nabla f\left(p_{N}(t)\right), w\right)\right|_{G_{\sigma}^{s^{*}}(\Omega)}^{2} & =\sum_{j=1}^{m}\left|\left(\nabla f_{j}\left(p_{N}(t)\right), w\right)\right|_{G_{\sigma}^{s^{*}}(\Omega)}^{2} \\
& \leqslant \sum_{j=1}^{m}\left(\sum_{i=1}^{m} C_{s^{*}} \cdot\left|\partial_{u_{i}} f_{j}\left(p_{N}(t)\right)\right|_{G_{\sigma}^{s^{*}}(\Omega)} \cdot\left|w_{i}\right|_{G_{\sigma}^{s^{*}}(\Omega)}\right)^{2} \\
& \leqslant C \cdot \sum_{j=1}^{m}\left[g_{j}^{\prime}\left(C_{s^{*}}\left|p_{N}(t)\right|_{G_{\sigma}^{s^{*}}(\Omega)}\right)\right]^{2} \cdot|w|_{G_{\sigma}^{s^{*}}(\Omega)}^{2},
\end{aligned}
$$

где $g_{j}^{\prime}(r)$ - производная функции $g_{j}(r)$, а константа $C$ не зависит от $N$. Наконец, те же рассуждения дают, что

$$
\begin{aligned}
& \left|\left(\nabla^{2} f\left(p_{N}(t)+\tau w\right) w, w\right)\right|_{G_{\sigma}^{s^{*}}(\Omega)}^{2} \\
& \quad \leqslant \sum_{j=1}^{m}\left(\sum_{l, k=1}^{m}\left|\partial_{u_{l} u_{k}}^{2} f_{j}\left(p_{N}(t)+\tau w\right)\right|_{G_{\sigma}^{s^{*}}(\Omega)} \cdot\left|w_{l} \cdot w_{k}\right|_{G_{\sigma}^{s^{*}}(\Omega)}\right)^{2} \\
& \quad \leqslant C \cdot \sum_{j=1}^{m}\left[g_{j}^{\prime \prime}\left(C_{s^{*}}\left|p_{N}(t)\right|_{G_{\sigma}^{s^{*}}(\Omega)}+C_{s^{*}}|w|_{G_{\sigma}^{s^{*}}(\Omega)}\right)\right]^{2} \cdot|w|_{G_{\sigma}^{s^{*}}(\Omega)}^{4},
\end{aligned}
$$

где $g_{j}^{\prime \prime}(r)$ - вторая производная функции $g_{j}(r)$ и константа $C$ не зависит от $N$. Таким образом, имеем, что

$$
\begin{aligned}
\left|Q_{N} f\left(p_{N}(t)+w\right)\right|_{G_{\sigma}^{s^{*}}(\Omega)}^{2} \leqslant & G_{0}\left(C_{s^{*}}\left|p_{N}(t)\right|_{G_{\sigma}^{s^{*}}(\Omega)}\right) \\
& +G_{1}\left(C_{s^{*}}\left|p_{N}(t)\right|_{G_{\sigma}^{s^{*}}(\Omega)}\right) \cdot|w|_{G_{\sigma}^{s^{*}}(\Omega)}^{2} \\
& +G_{2}\left(C_{s^{*}}\left|p_{N}(t)\right|_{G_{\sigma}^{s^{*}}(\Omega)}+C_{s^{*}}|w|_{G_{\sigma}^{s^{*}}(\Omega)}\right) \cdot|w|_{G_{\sigma}^{s^{*}}(\Omega)}^{4}
\end{aligned}
$$

Здесь

$$
G_{k}(r)=C \cdot \sum_{j=1}^{m}\left[g_{j}^{(k)}(r)\right]^{2}, \quad k=0,1,2
$$

где $g_{j}^{(k)}(r)$ - производная функции $g_{j}(r)$ порядка $k$ и константа $C$ не зависит от $N$. Так как $s^{*}<s+1$, то из (2.1) имеем, что

$$
\left|p_{N}(t)\right|_{G_{\sigma}^{s^{*}}(\Omega)} \leqslant e^{\sigma(1+N)}\left|p_{N}(t)\right|_{s+1} \leqslant e^{\sigma(1+N)} R_{0}, \quad t \geqslant t_{0}=t_{0}(B) .
$$

Поэтому если выбрать $\sigma=(1+N)^{-1}$, то получим

$\left|Q_{N} f\left(p_{N}(t)+w\right)\right|_{G_{\sigma}^{s^{*}}(\Omega)}^{2} \leqslant C_{0}+C_{1}|w|_{G_{\sigma}^{s^{*}}(\Omega)}^{2}+G_{2}\left(C_{2}+C_{s^{*}}|w|_{G_{\sigma}^{s^{*}}(\Omega)}\right) \cdot|w|_{G_{\sigma}^{s^{*}}(\Omega)}^{4}$

для всех $t \geqslant t_{0}$, где $C_{j}$ не зависят от $N, j=0,1,2$. Следовательно, из (2.9) вытекает, что

$$
\begin{aligned}
\left|Q_{N} f\left(p_{N}(t)+w\right)\right|_{G_{\sigma}^{s}(\Omega)}^{2} \leqslant & \left|Q_{N} f\left(p_{N}(t)+w\right)\right|_{G_{\sigma}^{s^{*}}(\Omega)}^{2} \\
\leqslant & C_{0}+C_{1}\left(1+N^{2}\right)^{-\delta}|w|_{G_{\sigma}^{s+1}(\Omega)}^{2} \\
& +\left(1+N^{2}\right)^{-2 \delta} G_{2}\left(C_{2}+C_{s^{*}}|w|_{G_{\sigma}^{s^{*}}(\Omega)}\right) \cdot|w|_{G_{\sigma}^{s+1}(\Omega)}^{4}
\end{aligned}
$$


где $\delta=s+1-s^{*}>0$. Поэтому из (2.6) и (2.10) имеем, что

$$
\frac{d}{d t} V(t)+\mu V(t) \leqslant C_{1}+C_{2}\left(1+N^{2}\right)^{-2 \delta}[V(t)]^{2} \cdot G_{2}\left(C_{3}+C_{4} \cdot[V(t)]^{1 / 2}\right)
$$

для всех достаточно больших $N$, где $\delta>0, \sigma=(1+N)^{-1}$, а константы $C_{j}>0$ не зависят от $N$. Ясно, что при достаточно больших $N$ функция

$$
F(V)=-\mu V+C_{1}+C_{2}\left(1+N^{2}\right)^{-2 \delta} V^{2} \cdot G_{2}\left(C_{3}+C_{4} \cdot V^{1 / 2}\right)
$$

имеет простой корень $V_{0} \equiv V_{0}(N)>0$ такой, что $F(V)>0$ при $V \in\left(0, V_{0}\right)$. Так как $V\left(t_{0}\right)=0$, то указанное свойство функции $F(V)$ и неравенство (2.11) дают, что $V(t) \leqslant V_{0}$ при $t \geqslant t_{0}$. Кроме того, так как $V_{0}(N) \rightarrow C_{1} \mu^{-1}$ при $N \rightarrow \infty$, то $V_{0}(N) \leqslant V_{0}^{*}$, где $V_{0}^{*}$ не зависит от $N$. Поэтому из $(2.6)$ имеем, что

$$
\left|\partial_{t} w(t)\right|_{G_{\sigma}^{s}(\Omega)}^{2}+|w(t)|_{G_{\sigma}^{s+1}(\Omega)}^{2} \leqslant \beta_{1}^{-1} V_{0}^{*} \equiv R^{2}, \quad t \geqslant t_{0}
$$

для достаточно больших $N$ с произвольным $0 \leqslant \sigma \leqslant(1+N)^{-1} \leqslant \sigma_{0}$. Из этого неравенства вытекает (2.5). Лемма 2.1 доказана.

Лемма 2.2. Пусть $u(t)=\left(u_{1}(t), \ldots, u_{m}(t)\right)$ - решение задачи (1.6), (2), (3) с начальными условиями $y=\left(u_{0} ; u_{1}\right) \in B$, где $B$ - некоторое ограниченное множество в $\mathbf{E}_{s}$ такое, что выполняется (2.1). Предположим, что $w(t)=$ $\left(w_{1}(t), \ldots, w_{m}(t)\right)$ - решение задачи (2.2)-(2.4). Тогда существует $N_{0}>0$ maкое, чmo

$$
\lim _{t \rightarrow \infty} \sup _{y \in B}\left\{\left\|\partial_{t}\left(Q_{N} u(t)-w(t)\right)\right\|_{s}^{2}+\left\|Q_{N} u(t)-w(t)\right\|_{s+1}^{2}\right\}=0
$$

для каждого $N \geqslant N_{0}$, где $Q_{N}=1-P_{N}$.

ДокАЗАТЕЛьство. Пусть $v(t)=Q_{N} u(t)-w(t)$. Тогда $v(t)-$ решение задачи

$$
\partial_{t}^{2} v(t, x)+\gamma \partial_{t} v(t, x)+\mathscr{L} v(t, x)+Q_{N}\left(f(u(t, x))-f\left(p_{N}(t)+w(t)\right)\right)=0
$$

в классе периодических функций:

$$
v\left(t, x+L e_{k}\right)=v(t, x), \quad k=1, \ldots, n, \quad x \in \mathbb{R}^{n}, \quad t>t_{0},
$$

с начальными условиями в момент $t_{0}$ :

$$
v\left(t_{0}\right)=Q_{N} u\left(t_{0}\right), \quad \partial_{t} v\left(t_{0}, x\right)=Q_{N} \partial_{t} u\left(t_{0}\right)
$$

Как и при доказательстве леммы 2.1 , при $0<\nu \leqslant \gamma / 2$ рассмотрим функционал

$$
W(t) \equiv W\left(\partial_{t} v(t), v(t)\right)=W_{0}\left(\partial_{t} v(t), v(t)\right)+\nu \cdot W_{1}\left(\partial_{t} v(t), v(t)\right),
$$

где

$$
W_{0}\left(\partial_{t} v(t), v(t)\right)=\frac{1}{2}\left(\left\|\partial_{t} v(t)\right\|_{s}^{2}+\sum_{i, j=1}^{m} d_{i j}\left(v_{i}(t), v_{j}(t)\right)_{s+1}\right)
$$

и

$$
W_{1}\left(\partial_{t} v(t), v(t)\right)=\left(v(t), \partial_{t} v(t)\right)_{s}+\frac{\gamma}{2}\|v(t)\|_{s}^{2}
$$


Как и ранее, ясно, что

$$
\beta_{1}\left(\left\|\partial_{t} v\right\|_{s}^{2}+\|v\|_{s+1}^{2}\right) \leqslant W(t) \leqslant \beta_{2}\left(\left\|\partial_{t} v\right\|_{s}^{2}+\|v\|_{s+1}^{2}\right)
$$

с положительными константами $\beta_{j}$, не зависящими от $\nu$. Стандартные вычисления дают, что

$$
\frac{d}{d t} W_{0}\left(\partial_{t} v, v\right) \leqslant-\frac{\gamma}{2}\left\|\partial_{t} v\right\|_{s}^{2}+\frac{1}{2 \gamma}\left\|Q_{N} \widetilde{f}(u, w)\right\|_{s}^{2}
$$

Здесь и далее полагаем

$$
\widetilde{f}(u, w)=f(u(t))-f\left(p_{N}(t)+w(t)\right) .
$$

Используя (2.9) при $\sigma=0$, имеем также, что

$$
\frac{d}{d t} W_{1}\left(\partial_{t} v, v\right) \leqslant\left\|\partial_{t} v\right\|_{s}^{2}-\left(d_{0}-\left(1+N^{2}\right)^{-1}\right)\|v\|_{s+1}^{2}+\frac{1}{4}\left\|Q_{N} \tilde{f}(u, w)\right\|_{s}^{2}
$$

Как и при доказательстве леммы 2.1, из (2.17)-(2.19) вытекает сушествование чисел $\nu>0$ и $\mu>0$ таких, что

$$
\frac{d}{d t} W(t)+\mu W(t) \leqslant-\frac{\nu d_{0}}{4}|v|_{s+1}^{2}+C \cdot\left\|Q_{N} \widetilde{f}(u, w)\right\|_{s}^{2}
$$

для всех достаточно больших $N$, где константа $C>0$ не зависит от $N$.

Оценим теперь величину $\left\|Q_{N} \widetilde{f}(u, w)\right\|_{s}^{2}$. Так как

$$
\tilde{f}(u, w)=\int_{0}^{1}(\nabla f(u-\tau v), v) d \tau
$$

имеем, что

$$
\left\|Q_{N} \tilde{f}(u, w)\right\|_{s}^{2} \leqslant\left\|Q_{N} \tilde{f}(u, w)\right\|_{s^{*}}^{2} \leqslant \int_{0}^{1}\|(\nabla f(u-\tau v), v)\|_{s^{*}}^{2} d \tau
$$

где $s^{*}$ - произвольное число со свойством $\max \{n / 2 ; s\}<s^{*}<s+1$. Из леммы 1.1 при $\sigma=0$ и из предположения (А3) вытекает, что

$\|(\nabla f(u-\tau v), v)\|_{s^{*}}^{2} \leqslant C \cdot\|\nabla f(u-\tau v)\|_{s^{*}}^{2}\|v\|_{s^{*}}^{2} \leqslant C_{1} \cdot \sum_{j=1}^{m}\left[g_{j}^{\prime}\left(C_{2}\|u-\tau v\|_{s^{*}}^{2}\right)\right]^{2}\|v\|_{s^{*}}^{2}$.

Из (2.1) и из (2.12) при $\sigma=0$ имеем, что

$$
\|u(t)-\tau v(t)\|_{s^{*}}^{2} \leqslant\|u(t)-\tau v(t)\|_{s+1}^{2} \leqslant C\left(R_{0}, R\right) \text { для всех } t \geqslant t_{0}=t_{0}(B) .
$$

Следовательно, используя (2.9), получаем, что

$\left\|Q_{N} \widetilde{f}(u, w)\right\|_{s}^{2} \leqslant C\left(R_{0}, R\right) \cdot\left(1+N^{2}\right)^{-\left(s+1-s^{*}\right)}\|v(t)\|_{s+1}^{2}$ для всех $t \geqslant t_{0}=t_{0}(B)$. 
Таким образом, из (2.20) следует существование числа $N_{0}$ такого, что

$$
\frac{d}{d t} W(t)+\mu W(t) \leqslant 0
$$

для всех $t \geqslant t_{0}=t_{0}(B), N \geqslant N_{0}$. Из этого соотношения вытекает, что $W(t) \leqslant$ $e^{-\mu\left(t-t_{0}\right)} W\left(t_{0}\right)$ для всех $t \geqslant t_{0}=t_{0}(B)$ и $N \geqslant N_{0}$. Поэтому, используя $(2.1)$ и (2.17), получаем (2.13). Лемма 2.2 доказана.

Леммы 2.1 и 2.2 позволяют завершить доказательство теоремы 1.1. Зафиксируем $N$ так, чтобы выполнялись утверждения лемм 2.1 и 2.2. Тогда существует $\sigma>0$ такое, что множество

$$
\mathscr{G}=\left\{\left(u_{0}, u_{1}\right) \in \mathbf{G}_{\sigma}^{s+1}(\Omega) \times \mathbf{G}_{\sigma}^{s}(\Omega):\left|u_{0}\right|_{G_{\sigma}^{s+1}(\Omega)}^{2}+\left|u_{1}\right|_{G_{\sigma}^{s}(\Omega)}^{2} \leqslant \bar{R}^{2}\right\}
$$

является равномерно притягиваюшим для динамической системњ $\left(S_{t}, \mathbf{E}_{s}\right)$. Здесь $\bar{R}^{2}=e \cdot R_{0}^{2}+R^{2}$, а $R_{0}$ и $R$ - константы из (2.1) и (2.5). Поэтому из стандартных теорем о существовании аттрактора для асимптотически компактного случая (см, например, [9] или [11]) следует, что $\mathscr{A} \subset \mathscr{G}$.

ЗАмЕчАнИЕ 2.1. Представленный выше метод может быть также применен к другим эволюционным уравнениям второго порядка по времени. Например, можно доказать утверждение, аналогичное теореме 1.1, для уравнения (1) с бигармоническим оператором $\Delta^{2}$ вместо $-\Delta$. В этом случае допустима зависимость нелинейного члена $f(u)$ от пространственных производных первого порядка.

\section{§3. Определяющие узлы $(n=1)$}

В этом параграфе доказана теорема 1.2 о существовании определяющих узлов для задачи (1)-(3) в случае одной пространственной переменной.

Начнем со следующей простой леммы.

ЛЕмма 3.1. Предположим, что $x_{1}$ и $x_{2}$ - узль такие, что $0 \leqslant x_{1}<x_{2} \leqslant L$. Пусть $u^{1}(t, x)$ и $u^{2}(t, x)$ - два решения задачи (1), (2) при $n=1$ из класса

$$
\mathscr{W}_{2}=C^{1}\left(0,+\infty ; \mathbf{H}_{\mathrm{per}}^{2}(0, L)\right) \cap C\left(0,+\infty ; \mathbf{H}_{\mathrm{per}}^{3}(0, L)\right)
$$

такие, что

$$
\left\|\partial_{t} u^{k}(t)\right\|_{2}^{2}+\left\|u^{k}(t)\right\|_{3}^{2} \leqslant R^{2} \quad n p u \quad t \geqslant t_{0}, \quad k=1,2,
$$

для некоторых $R>0$ и $t_{0} \geqslant 0$. Тогда существуют числа $\Delta_{0} \equiv \Delta_{0}(R)>0 u$ $\mu>0$ такие, что при условии $0<x_{2}-x_{1}<\Delta_{0}$ для разности $u(t) \equiv u(t, x)=$ $u^{1}(t, x)-u^{2}(t, x)$ при всех $t \geqslant s \geqslant t_{0}$ имеем оценку

$$
\begin{aligned}
\left\|\partial_{t} u(t)\right\|_{\Delta}^{2}+ & \|u(t)\|_{1, \Delta}^{2} \leqslant c_{1} e^{-\mu(t-s)} \\
& +c_{2} \max _{l=1,2}\left|u\left(t, x_{l}\right)\right|+c_{3} \cdot \int_{s}^{t} e^{-\mu(t-\tau)} \cdot \max _{l=1,2}\left|u\left(\tau, x_{l}\right)\right| d \tau,
\end{aligned}
$$

где $c_{j}$ - положительные константьи, зависящие от $R$. Здесь и далее используются обозначения: $\Delta=\left\{x: x_{1} \leqslant x \leqslant x_{2}\right\},|\Delta|=x_{2}-x_{1} u$

$$
\|w\|_{\Delta}^{2}=\int_{x_{1}}^{x_{2}}|w(x)|^{2} d x, \quad\|w\|_{1, \Delta}^{2}=\|w\|_{\Delta}^{2}+\left\|\partial_{x} w\right\|_{\Delta}^{2} .
$$


ДокАЗАТЕЛЬСтво. Воспользуемся тем же подходом, что и при доказательстве лемм 2.1 и 2.2. Из (1) имеем, что функция $u(t, x)$ удовлетворяет уравнению

$\partial_{t}^{2} u(t, x)+\gamma \partial_{t} u(t, x)+\mathscr{L} u(t, x)+f\left(u^{1}(t, x)\right)-f\left(u^{2}(t, x)\right)=0, \quad x \in(0, L), t>0$.

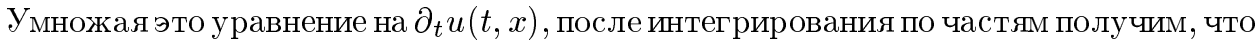

$$
\begin{aligned}
\frac{d}{d t} W_{0}^{\Delta}(t) \leqslant & -\frac{\gamma}{2}\left\|\partial_{t} u(t)\right\|_{\Delta}^{2} \\
& +\eta\left(t, x_{2}\right)-\eta\left(t, x_{1}\right)+(2 \gamma)^{-1} \int_{\Delta}\left|f\left(u^{1}(t, x)\right)-f\left(u^{2}(t, x)\right)\right|^{2} d x
\end{aligned}
$$

где

$$
W_{0}^{\Delta}(t)=\frac{1}{2}\left(\left\|\partial_{t} u(t)\right\|_{\Delta}^{2}+\sum_{i, j=1}^{m} d_{i j} \int_{\Delta}\left[\partial_{x} u_{i}(t, x) \cdot \partial_{x} u_{j}(t, x)+u_{i}(t, x) \cdot u_{j}(t, x)\right] d x\right)
$$

и

$$
\eta(t, x)=\sum_{i, j=1}^{m} d_{i j} \partial_{x} u_{i}(t, x) \cdot \partial_{t} u_{j}(t, x)
$$

Для функции

$$
W_{1}^{\Delta}(t)=\int_{\Delta}\left(u(t, x), \partial_{t} u(t, x)\right)_{\Delta} d x+\frac{\gamma}{2}\|u(t)\|_{\Delta}^{2}
$$

имеем также, что

$$
\begin{aligned}
\frac{d}{d t} W_{1}^{\Delta}(t)= & \left\|\partial_{t} u\right\|_{\Delta}^{2}+\sum_{i, j=1}^{m} d_{i j} \int_{\Delta}\left(\left(\partial_{x}^{2}-1\right) u_{i}(t, x), u_{j}(t, x)\right) d x \\
& -\int_{\Delta}\left(f\left(u^{1}(t, x)\right)-f\left(u^{2}(t, x)\right), u(t, x)\right) d x \\
\leqslant & \left\|\partial_{t} u\right\|_{\Delta}^{2}-\frac{d_{0}}{2}\|u\|_{1, \Delta}^{2} \\
& +C_{1}\left\|f\left(u^{1}(t)\right)-f\left(u^{2}(t)\right)\right\|_{\Delta}^{2}+C_{2} \sum_{l=1,2}\left|u\left(\tau, x_{l}\right)\right| \cdot\left|\partial_{x} u\left(\tau, x_{l}\right)\right| .
\end{aligned}
$$

Поэтому, как и ранее, при достаточно малых $\mu>0$ и $\nu>0$ функционал $W^{\Delta}(t)=$ $W_{0}^{\Delta}(t)+\nu \cdot W_{1}^{\Delta}(t)$ обладает свойством

$$
\begin{aligned}
\frac{d}{d t} W^{\Delta}(t)+\mu W^{\Delta}(t) \leqslant & -\frac{\nu d_{0}}{4}\|u\|_{1, \Delta}^{2}+C_{1}\left\|f\left(u^{1}(t)\right)-f\left(u^{2}(t)\right)\right\|_{\Delta}^{2} \\
& +C_{2} \max _{l=1,2}\left|u\left(t, x_{l}\right)\right| \cdot\left|\partial_{x} u\left(t, x_{l}\right)\right|+\eta\left(t, x_{2}\right)-\eta\left(t, x_{1}\right) .
\end{aligned}
$$

Так как $H_{\mathrm{per}}^{1}(0, L)$ непрерьвно вложено в $C([0, L])$, то из $(3.1)$ легко видеть, что

$$
\left|\partial_{x} u\left(t, x_{l}\right)\right| \leqslant C\|u(t)\|_{2} \leqslant C_{R}
$$


И

$$
\begin{aligned}
\left\|f\left(u^{1}(t)\right)-f\left(u^{2}(t)\right)\right\|_{\Delta}^{2} & \leqslant C_{R} \cdot\left\|u^{1}(t)-u^{2}(t)\right\|_{\Delta}^{2} \\
& \leqslant C_{R} \cdot\left(\left|u\left(t, x_{1}\right)\right|^{2}+|\Delta|^{2} \cdot\|u\|_{1, \Delta}^{2}\right) .
\end{aligned}
$$

Поэтому при достаточно малых $|\Delta|$ имеем, что

$$
\frac{d}{d t} W^{\Delta}(t)+\mu W^{\Delta}(t) \leqslant C_{R} \max _{l=1,2}\left|u\left(t, x_{l}\right)\right|+\eta\left(t, x_{2}\right)-\eta\left(t, x_{1}\right) .
$$

Из этого неравенства вытекает, что

$$
\begin{aligned}
W^{\Delta}(t) \leqslant C_{R} \cdot e^{-\mu(t-s)}+C_{R} \int_{s}^{t} e^{-\mu(t-\tau)} \max _{l=1,2}\left|u\left(\tau, x_{l}\right)\right| d \tau \\
\quad+\int_{s}^{t} e^{-\mu(t-\tau)}\left[\eta\left(\tau, x_{2}\right)-\eta\left(\tau, x_{1}\right)\right] d \tau .
\end{aligned}
$$

Однако интегрирование по частям дает, что

$$
\begin{aligned}
\int_{s}^{t} e^{-\mu(t-\tau)} \eta(\tau, x) d \tau \\
=\sum_{i, j=1}^{m} d_{i j} \int_{s}^{t} e^{-\mu(t-\tau)} \partial_{x} u_{i}(\tau, x) \cdot \partial_{t} u_{j}(\tau, x) d \tau \\
=\sum_{i, j=1}^{m} d_{i j}\left\{\partial_{x} u_{i}(t, x) \cdot u_{j}(t, x)-e^{-\mu(t-s)} \partial_{x} u_{i}(s, x) \cdot u_{j}(s, x)\right\} \\
\quad-\sum_{i, j=1}^{m} d_{i j}\left\{\mu \int_{s}^{t} e^{-\mu(t-\tau)} \partial_{x} u_{i}(\tau, x) \cdot u_{j}(\tau, x) d \tau\right. \\
\left.\quad+\int_{s}^{t} e^{-\mu(t-\tau)} \partial_{x t}^{2} u_{i}(\tau, x) \cdot u_{j}(\tau, x) d \tau\right\}
\end{aligned}
$$

Поэтому из (3.1) имеем, что

$$
\left|\int_{s}^{t} e^{-\mu(t-\tau)} \eta(\tau, x) d \tau\right| \leqslant C_{R} \cdot\left\{e^{-\mu(t-s)}+|u(t, x)|+\int_{s}^{t} e^{-\mu(t-\tau)}|u(\tau, x)| d \tau\right\} .
$$

Из этого неравенства и из (3.3) вытекает (3.2).

ЛЕмма 3.2. Предположим, что $n=1$ и выполнены предположения (A1)-(А3) при $s=0$. Тогда существует $R>0$ такое, что для любого решения $u(t, x)$ задачи (1), (2), лежащего в классе $\mathscr{W}_{2}$, имеем, что

$$
\left\|\partial_{t} u(t)\right\|_{2}^{2}+\|u(t)\|_{3}^{2} \leqslant R^{2}, \quad t \geqslant t_{0}(u) .
$$


ДокАЗАТЕльство. Прежде всего отметим, что существование глобального аттрактора (см. (А2) с $s=0)$ дает, что

$$
\left\|\partial_{t} u(t)\right\|^{2}+\|u(t)\|_{1}^{2} \leqslant R_{0}^{2}, \quad t \geqslant \bar{t}_{0}(u)
$$

с некоторьми $R_{0}$ и $\bar{t}_{0}(u)(\mathrm{cp} .(2.1))$. Кроме того, функция $v(t, x)=\partial_{t} u(t, x)$ является решением задачи

$$
\partial_{t}^{2} v(t)+\gamma \partial_{t} v(t)+\mathscr{L} v(t)=F(t) \equiv-\left(\nabla f(u(t)), \partial_{t} u(t)\right), \quad t>0,
$$

с периодическими условиями и с начальньми данньми:

$$
v(0, x)=u_{1}, \quad \partial_{t} v(0, x)=-\gamma u_{1}-\mathscr{L} u_{0}-f\left(u_{0}\right)+h .
$$

Так как

$$
\begin{aligned}
\|F(t)\| & =\left\|\left(\nabla f(u(t)), \partial_{t} u(t)\right)\right\| \leqslant\|\nabla f(u(t))\|_{\mathbf{L}^{\infty}} \cdot\left\|\partial_{t} u(t)\right\| \\
& \leqslant C \cdot\left(\sum_{j=1}^{m}\left[g_{j}^{\prime}\left(\max _{(0, L)}|u(t, x)|\right)\right]^{2}\right)^{1 / 2}\left\|\partial_{t} u(t)\right\|
\end{aligned}
$$

и так как $H_{\mathrm{per}}^{1}(0, L)$ непрерывно вложено в $C([0, L])$, то из $(3.5)$ следует, что

$$
\limsup _{t \rightarrow+\infty}\|F(t)\| \leqslant C\left(R_{0}\right)
$$

Поэтому стандартные рассуждения, аналогичные приведенным в леммах 2.1 или 2.2 , позволяют получить соотношение

$$
\left\|\partial_{t t}^{2} u(t)\right\|^{2}+\left\|\partial_{t} u(t)\right\|_{2}^{2}+\|u(t)\|_{3}^{2} \leqslant R_{1}^{2}, \quad t \geqslant t_{1}(u),
$$

где $R_{1}$ зависит от $R_{0}$ и от параметров уравнения (1). Используя это соотношение и дифференцируя (3.6) по $t$, можно применить тот же, что и ранее, метод и получить (3.4). Аналогичные и более подробные рассуждения для общего случая эволюционных уравнений второго порядка по времени могут быть найдены в [14].

Теперь мы можем доказать теорему 1.2.

Пусть выполняется $(1.9)$ и $0<x_{2}-x_{1}<\Delta_{0}$, где $\Delta_{0}-$ то же самое число, что и в лемме 3.1. Тогда леммы 3.1 и 3.2 дают, что

$$
\lim _{t \rightarrow \infty}\left\{\left\|\partial_{t} u(t)-\partial_{t} u^{*}(t)\right\|_{\Delta}^{2}+\left\|u(t)-u^{*}(t)\right\|_{1, \Delta}^{2}\right\}=0
$$

где $\Delta=\left(x_{1}, x_{2}\right)$. Предположим, что (1.10) не верно. В этом случае так как

$$
\operatorname{dist}_{\mathbf{E}_{0}}\left(\left(u(t) ; \partial_{t} u(t)\right), \mathscr{A}\right) \rightarrow 0, \quad t \rightarrow \infty,
$$

для любого решения $u(t) \in \mathscr{W}_{0}$, то существует последовательность $\left\{t_{n}\right\}$ и элементы $a=a(x)$ и $a^{*}=a^{*}(x)$ из $\mathscr{A}$ такие, что

$$
\lim _{n \rightarrow \infty}\left(\left\|\partial_{t} u\left(t_{n}\right)-\partial_{t} u^{*}\left(t_{n}\right)\right\|^{2}+\left\|u\left(t_{n}\right)-u^{*}\left(t_{n}\right)\right\|_{1}^{2}\right)>0
$$

и

$$
\lim _{n \rightarrow \infty}\left\|\left(u\left(t_{n}\right) ; \partial_{t} u\left(t_{n}\right)\right)-a\right\|_{\mathbf{E}_{0}}=0, \quad \lim _{n \rightarrow \infty}\left\|\left(u^{*}\left(t_{n}\right) ; \partial_{t} u^{*}\left(t_{n}\right)\right)-a^{*}\right\|_{\mathbf{E}_{0}}=0 .
$$

Из (3.8) вытекает, что $a(x)=a^{*}(x)$ при $x \in \Delta=\left(x_{1}, x_{2}\right)$. Так как элементы аттрактора $\mathscr{A}$ являются вещественно-аналитическими функциями по пространственной переменной (см. теорему 1.1 ), то имеем, что $a(x)=a^{*}(x)$ для всех $x \in(0, L)$. Это противоречит соотношению (3.9). Теорема 1.2 доказана. 
ЗАМЕЧАНИЕ 3.1. Использую интерполяционные неравенства для соболевских норм и некоторые дополнительные рассуждения, из (3.4) можно извлечь более сильную сходимость к нулю величины $u(t)-u^{*}(t)$. А именно, в условиях теоремы 1.2 можно вместо (1.10) доказать, что

$$
\lim _{t \rightarrow \infty}\left\{\left\|\partial_{t}\left(u(t)-u^{*}(t)\right)\right\|_{2}^{2}+\left\|u(t)-u^{*}(t)\right\|_{3}^{2}\right\}=0 .
$$

Отметим также, что из теоремы 1.1 и леммы 3.1 вытекает следуюшее свойство любых двух траекторий $y^{k}(t)=\left(u^{k}(t, x) ; \partial_{t} u^{k}(t, x)\right), k=1,2$, лежащих в глобальном аттракторе $\mathscr{A}$ задачи (1)-(3) в случае одной пространственной переменной: если $x_{1}$ и $x_{2}$ - достаточно близкие друг к другу узлы на интервале $(0, L)$ такие, что

$$
u^{1}\left(t, x_{l}\right)=u^{2}\left(t, x_{l}\right) \quad \text { для всех } t \in(-\infty,+\infty), \quad l=1,2,
$$

TO

$$
u^{1}(t, x)=u^{2}(t, x) \text { для всех } t \in(-\infty,+\infty), \quad x \in(0, L) .
$$

Действительно, из теоремы 1.1 вытекает, что

$$
\left\|\partial_{t} u(t)\right\|_{q}^{2}+\|u(t)\|_{q+1}^{2}<R_{q}^{2} \text { для всех } t \in(-\infty,+\infty), \quad q=0,1, \ldots
$$

Здесь $\left(u(t) ; \partial_{t} u(t)\right)$ - траектория, лежащая в глобальном аттракторе $\mathscr{A}$. Поэтому можно применить лемму 3.1 при каждом $t \geqslant s>-\infty$. Следовательно, (3.2) и (3.10) дают,что

$$
\left\|\partial_{t} u(t)\right\|_{\Delta}^{2}+\|u(t)\|_{1, \Delta}^{2} \leqslant c_{1} e^{-2 \mu(t-s)} \text { для любых } t \geqslant s,
$$

где $u(t, x)=u^{1}(t, x)-u^{2}(t, x)$. Поэтому если зафиксировать $t$ и устремить $s \rightarrow-\infty$, то получим, что $u(t, x)=0$ для всех $t \in(-\infty,+\infty)$ и $x \in\left(x_{1}, x_{2}\right)$. Отсюда вытекает (3.11), потому что $y^{1}(t), y^{2}(t) \in \mathscr{A}$ - вешественно-аналитические функции по пространственной переменной (см. теорему 1.1).

ЗАмЕчАниЕ 3.2. Утверждение, аналогичное теореме 1.2, может быть также получено для решений $u$ и $u^{*}$ из $\mathscr{W}_{1}$ (вместо $\mathscr{W}_{2}$ ). В этом случае нужны дополнительные определяющие функционалы. Например, можно доказать, что из (1.9) и одного из дополнительных предположений

$$
\lim _{t \rightarrow \infty} \max _{l=1,2} \sum_{j=1}^{m}\left|\partial_{x} u_{j}\left(t, x_{l}\right)-\partial_{x} u_{j}^{*}\left(t, x_{l}\right)\right|=0
$$

или

$$
\lim _{t \rightarrow \infty} \max _{l=1,2} \sum_{j=1}^{m}\left|\partial_{t} u_{j}\left(t, x_{l}\right)-\partial_{t} u_{j}^{*}\left(t, x_{l}\right)\right|=0
$$

вытекает (1.10). Для доказательства нужно только заметить, что (3.3) остается в силе для разности $u(t)$ любых двух решений $u^{1}(t)$ и $u^{2}(t)$ из $\mathscr{W}_{1}$, обладаюших свойством

$$
\left\|\partial_{t} u^{k}(t)\right\|_{1}^{2}+\left\|u^{k}(t)\right\|_{2}^{2} \leqslant R^{2} \text { при } t \geqslant t_{0}, \quad k=1,2 .
$$

Это позволяет для указанных случаев получить неравенства, аналогичные (3.2). После этого достаточно воспользоваться (3.7) и повторить те же самые рассуждения, что и в теореме 1.2 . 


\section{§4. Примеры}

В этом параграфе кратко описаны несколько ситуаций, в которых могут быть использованы теоремы 1.1 и 1.2. Ниже во всех примерах предполагается выполненным условие (2) пространственной периодичности.

ПримеР 4.1. Пусть $n \leqslant 3, m \geqslant 1$. Рассмотрим систему связанных волновых уравнений с демпфированием

$$
\begin{gathered}
\partial_{t}^{2} u_{j}(t, x)+\gamma \partial_{t} u_{j}(t, x)-\Delta u_{j}(t, x)+\lambda\left[u_{j}(t, x)\right]^{3}+\sum_{i=1}^{m} a_{j i} u_{i}(t, x)=h_{j}(x), \\
x \in \mathbb{R}^{n}, \quad t>0, \quad j=1, \ldots, m
\end{gathered}
$$

где $\left\{a_{j i}\right\}$ - матрица с постоянньми элементами, $\gamma$ и $\lambda$ - положительные параметры. Используя стандартные методы (см., например, [15], где рассмотрен случай $m=1$ ), легко обнаружить, что задача $(4.1),(2),(3)$ корректно разрешима в класс $\mathscr{W}_{0}$ (классы $\mathscr{W}_{s}$ определяются формулой (1.7)). Используя подход, представленный в [14], можно доказать (ср. замечание 1.1), что эта задача корректно разрешима в каждом классе $\mathscr{W}_{k}, k=0,1, \ldots$, а эволюционный оператор $S_{t}$ обладает глобальным аттрактором в пространстве $\mathbf{E}_{1}=\mathbf{H}_{\text {per }}^{2}(\Omega) \times \mathbf{H}_{\text {per }}^{1}(\Omega)$. Если матрица $\left\{a_{j i}\right\}$ является симметрической (это всегда так при $m=1$ ), то задача имеет функционал Ляпунова и поэтому в случае общего положения аттрактор имеет регулярную структуру (см., например, [10], [11] или [12]). Таким образом, выполняются гипотезы (А1)-(А3) с $s=1$ при условии, что $h_{j}(x)$ лежит в некотором классе Жевре. Эти же рассуждения и вьводы остаются в силе, если вместо (4.1) рассмотреть систему

$\partial_{t}^{2} u_{j}(t, x)+\gamma \partial_{t} u_{j}(t, x)-\Delta u_{j}(t, x)+\lambda u_{j}(t, x) \cdot \sum_{i=1}^{m}\left[u_{i}(t, x)\right]^{2}+\sum_{i=1}^{m} a_{j i} u_{i}(t, x)=h_{j}(x)$,

где $x \in \mathbb{R}^{n}, t>0, j=1, \ldots, m$.

ПримеР 4.2. В случае одной пространственной переменной допустимы нелинейности более общего вида. Например, можно рассмотреть следующую систему

$$
\begin{gathered}
\partial_{t}^{2} u_{j}(t, x)+\gamma \partial_{t} u_{j}(t, x)-\partial_{x x} u_{j}(t, x)+f\left(u_{j}(t, x)\right)+\sum_{i=1}^{m} a_{j i} u_{i}(t, x)=h_{j}(x), \\
x \in \mathbb{R}^{1}, \quad t>0, \quad j=1, \ldots, m .
\end{gathered}
$$

Здесь $\gamma>0, m \geqslant 1,\left\{a_{j i}\right\}$ - матрица с постоянными элементами и $f(u)$ - полином нечетной степени с положительным старшим коэффициентом. Легко видеть (cp. [14] и [12]), что когда $h_{j}(x)$ - функции класса Жевре, условия (A1)-(А3) выполняются с $s=0$. В этом случае применимы обе теоремы 1.1 и 1.2.

ПримеР 4.3. Можно также рассмотреть несколько уравнений синус-Гордона с демпфированием. Они используются при моделировании динамики контактов Джозефсона, возбуждаемых источником тока (ссылки и комментарии см., например, в [12]). Рассмотрим сначала скалярное уравнение синус-Гордона

$$
\partial_{t}^{2} u+\gamma \partial_{t} u-\Delta u+\beta u+\lambda \sin u=h(x), \quad x \in \mathbb{R}^{n}, \quad t>0 .
$$


Здесь $n \leqslant 3$ а $\gamma, \beta$ и $\lambda$ - положительные константы. Ясно, что функция $f(u)=$ $\lambda \sin u$ обладает свойствами, перечисленньми в (А3). Как и в приведенных ранее примерах, свойства (A1) и (А2) могут быть установлены методом, представленным в [14] и [12]. Те же заключения справедливы и для пары связанных уравнений синус-Гордона:

$$
\begin{aligned}
& \partial_{t}^{2} u_{1}+\gamma \partial_{t} u_{1}-\Delta u_{1}+\beta u_{1}+\lambda \sin u_{1}+\alpha\left(u_{1}-u_{2}\right)=h_{1}(x), \\
& \partial_{t}^{2} u_{2}+\gamma \partial_{t} u_{2}-\Delta u_{2}+\beta u_{2}+\lambda \sin u_{2}-\alpha\left(u_{1}-u_{2}\right)=h_{2}(x),
\end{aligned}
$$

а также для системы (см. в [12]):

$$
\begin{aligned}
& \partial_{t}^{2} u_{1}+\gamma \partial_{t} u_{1}-\Delta u_{1}+\beta u_{1}+\lambda \sin \left(u_{1}+u_{2}\right)=h_{1}(x) \\
& \partial_{t}^{2} u_{2}+\gamma \partial_{t} u_{2}-\Delta u_{2}+\beta u_{2}+\lambda \sin \left(u_{1}-u_{2}\right)=h_{2}(x)
\end{aligned}
$$

Отметим, что в приведенных выше уравнениях можно положить $\beta=0$. Однако в этом случае необходимо слегка видоизменить фазовое пространство. Нужно его факторизовать (подробности см. в [12]) для того, чтобы отождествить решения $u(t, x)$ и $u^{*}(t, x)$, обладающие свойством $u(t, x)-u^{*}(t, x)=2 \pi k, k \in \mathbb{Z}$.

\section{Список литературы}

1. Foias C., Temam R. Gevrey class regularity for the solutions of the Navier-Stokes equations // J. Funct. Anal. 1989. V. 87. P. 359-369.

2. Promislow $K$. Time analyticity and Gevrey regularity for solutions of a class of dissipative partial differential equations // Nonlinear Anal. 1991. V. 16. P. 959-980.

3. Kukavica I. On the number of determining nodes for the Ginzburg-Landau equation // Nonlinearity. 1992. V. 5. P. 997-1006.

4. Foias C., Kukavica I. Determining nodes for the Kuramoto-Sivashinsky equation // J. Dynam. Differential Equations. 1995. V. 7. P. 365-373.

5. Ferrari A.B., Titi E.S. Gevrey regularity of solutions of a class of analytic nonlinear parabolic equations // Comm. Partial Differential Equations. 1998. V. 2. P. 1-16.

6. Goubet $O$. Regularity of the attractor for a weakly damped driven nonlinear Scrödinger equation // Appl. Anal. 1996. V. 60. P. 99-119.

7. Oliver M., Titi E.S. Analyticity of the attractor and the number of determining nodes for a weakly damped driven nonlinear Scrödinger equation // Indiana Univ. Math. J. 1998. V. 47. P. 49-73.

8. Чуешов И. Д. Теория функционалов, однозначно определяющих асимптотическую динамику бесконечномерных диссипативных систем // УМН. 1998. Т. 53. № 4. С. 77-124.

9. Ладъ женская $О$. А. О нахождении минималњных глобалњных аттракторов для уравнения Навье-Стокса и других уравнений с частнњми производными // УМН. 1987. Т. 42. №6. C. $25-60$.

10. Бабин А. В., Вишик М. И. Аттракторы эволюционных уравнений. М.: Наука, 1989.

11. Hale J. K. Asymptotic behavior of dissipative systems. Providence: Amer. Math. Soc., 1988.

12. Temam $R$. Infinite-dimensional dynamical systems in mechanics and physics. New York: Springer-Verlag, 1988.

13. Чуешов И. Д. Глобальные аттракторы в нелинейных задачах математической физики // УМН. 1993. Т. 48. №3. С. 135-162.

14. Ghidaglia J.-M., Temam R. Regularity of the solutions of second order evolution equations and their attractors // Ann. Scuola Norm. Sup. Pisa Cl. Sci. (4). 1987. V. 14. P. 485-511.

15. Lions J.-L. Quelques methodes de resolution de problemes aus limites non lineaires. Paris: Dunod, 1969. 